\title{
High-resolution wavefront-guided surface ablation with corneal cross-linking in ectatic corneas: a pilot study
}

This article was published in the following Dove Press journal:

Clinical Ophthalmology

3 October 2017

Number of times this article has been viewed

\section{Gustavo E Tamayo \\ Claudia Castell \\ Pilar Vargas \\ Eduardo Polania \\ Juliana Tamayo}

Bogota Laser Ocular Surgery Center, Bogota, Colombia
Correspondence: Gustavo E Tamayo Bogota Laser Ocular Surgery Center, Calle II 3 \# 7 45 Torre B Suite 906,

Bogota, Colombia

Tel +57 I6 292849

Fax +57 16292905

Email centrogustavotamayo@gmail.com
Purpose: A preliminary study to evaluate the outcomes of high-resolution wavefront-guided (HRWG) photorefractive keratectomy (PRK) with simultaneous corneal cross-linking (CXL) in ectasia eyes.

Methods: Sixteen eyes of 11 patients (mean age 31.9 \pm 9.8 years; range: $15-48$ years) with keratoconus or post-laser-assisted in situ keratomileusis ectasia underwent HRWG PRK with simultaneous CXL. Manifest refraction, uncorrected (UDVA) and corrected distance visual acuity (CDVA), and keratometric and aberrometric outcomes are reported at 12 months.

Results: Significant improvement was observed postoperatively in visual acuity, refraction, and keratectomy in all eyes. At postoperative 12 months, $87.5 \%$ eyes were within $\pm 1.0 \mathrm{D}$ of attempted correction and $81.25 \%$ of eyes had a postoperative UDVA of $20 / 32$ or better. A gain of 2 or more lines of CDVA was observed in $12.5 \%(2 / 16)$ of eyes and there was no change in CDVA lines in 25\% (4/16) eyes. A substantial reduction in higher-order aberrations was observed in all eyes postoperatively; however, the improvement was not statistically significant.

Conclusion: The outcomes of HRWG PRK in ectasia eyes with estimated residual stromal bed thickness of at least $350 \mu \mathrm{m}$ (without epithelium) are promising at postoperative 1 year and provide surgeons with a valuable tool to improve vision with a high degree of refractive predictability.

Keywords: wavefront ablation, corneal cross linking, irregular astigmatism, keratoconus, ectasia

\section{Introduction}

Correction of the irregular corneal shape, plus correction of higher-order aberrations (HOAs) and low-order aberrations (spherocylindrical errors) is a goal that is difficult to achieve in eyes with irregular astigmatism. Conventional aberrometers and wavefrontguided (WG) excimer laser ablations have been successfully used to capture and treat HOAs in corneas with regular corneal surface anatomy (eg, eyes without any type of treatment or disease). However, in eyes with irregular corneas such as eyes with keratoconus or post-laser-assisted in situ keratomileusis (LASIK) ectasia, conventional wavefront aberrometers have often not been able to provide sufficient or sufficiently repeatable data with which to guide treatments. ${ }^{1}$

Topography-guided (TG) laser ablation may be valuable in this situation by treating the corneal shape rather than physiological changes. Corneal ectasia, whether natural (keratoconus) or iatrogenic (post-LASIK ectasia), has been successfully managed by TG ablation procedures. ${ }^{2-5}$ With the advent of corneal cross-linking (CXL), which 
increases the biomechanical strength of the cornea, TG photorefractive keratectomy (PRK) with simultaneous/ sequential CXL has gained widespread acceptance. ${ }^{6-9}$

TG treatment is essentially an anatomical treatment to correct the anterior corneal shape. In ectasia eyes, this alone may substantially reduce refractive error. However, TG treatment does not take into account the refractive component, the posterior corneal anatomy, or any internal aberrations. ${ }^{1}$ Based on the surface irregularity being corrected, ${ }^{1}$ TG anatomical shape change induces an unknown spherical component of the correction. Therefore, some ectasia eyes may be left with significant refractive error after TG treatment, thereby decreasing its refractive predictability. ${ }^{1,4} \mathrm{WG}$ ablations change the shape of the cornea and at the same time address the refractive error, taking into account all optical aberrations (higher- and lower order) including those from posterior cornea or those arising intraocularly. If an accurate wavefront can be captured for eyes with irregular corneal shape, a WG procedure has the potential to not only regularize the anterior corneal surface, but also provide better refractive outcomes.

iDesign (Abbott Medical Optics, Inc., Santa Ana, CA, USA) is a new-generation aberrometer, which like previous generation aberrometers is based on Hartmann-Shack WaveScan aberrometer, but has higher resolution and improved software that allows it to analyze up to 1,257 wavefront data points from a $7.0-\mathrm{mm}$ pupil. ${ }^{10,11}$ The device relies on Fourier algorithm for data reconstruction. ${ }^{10}$ In addition to wavefront aberrometry, the device also captures autorefraction, topography, keratectomy, and pupillometry and provides absolute registration of eye motion and pupil shift, technical specifications of which have been validated previously in normal eyes undergoing high-resolution wavefrontguided (HRWG) refractive surgeries. ${ }^{11-13}$

This retrospective, pilot study was aimed at evaluating the outcomes of the off-label use of HRWG surface ablation with CXL in mild cases of ectasia and irregular astigmatism due to primary keratoconus and post-LASIK ectasia. Results may serve as the base for a larger study with longer follow-up.

\section{Methods}

This retrospective chart review studied all consecutive cases of irregular astigmatism due to keratoconus or postLASIK ectasia, who had undergone HRWG PRK (iDesign, Abbott Medical Optics, Inc., Santa Ana, CA, USA) with simultaneous CXL between June 2013 and May 2015 at Bogota Laser Ocular Surgery Center, Bogota, Colombia. The study was approved by the Institutional Review Board of Bogota Laser Ocular Surgery Center. Eyes were excluded if they were lost to follow-up prior to the 1-year postoperative visit or if they had poor vision due to other conditions in addition to ectasia. Sixteen eyes of 11 patients (seven women, four men with mean age $31.9 \pm 9.8$ years; age range: 15-48 years) met the inclusion criteria. Of these 16 eyes, 10 eyes had keratoconus $(62.5 \%)$ and six eyes had postLASIK ectasia (37.5\%). The diagnosis of keratoconus/postLASIK ectasia was based on Pentacam parameters. The Pentacam descriptors analyzed were keratectomy (steep, flat, and mean), pachymetry (central, thinnest, and apex), corneal thickness progression indices, anterior and posterior elevation maps, anterior surface topometric indices, normalized deviation indices, and overall deviation of normality (Belin/Ambrosio Enhanced Ectasia Display-Final D index). Presence of irregular astigmatism was evaluated by assessing contact lens-corrected visual acuity using rigid gas permeable lenses and ascertaining improvement over spectacle-corrected visual acuity.

All patients had a complete preoperative ophthalmic examination including manifest and cycloplegic refraction, uncorrected (UDVA) and corrected distance visual acuity (CDVA) testing, slit-lamp anterior segment examination, corneal topography and anterior segment imaging using the Pentacam-HR system (Oculus Optikgeräte Inc., Wetzlar, Germany), ocular aberrometry using the iDesign aberrometer, applanation tonometry, and fundoscopy. All patients received a complete explanation of the surgery and its risks and benefits and signed an informed consent prior to the surgery. As a standard clinical protocol, the estimated residual stromal bed thickness must be at least $350 \mu \mathrm{m}$ for consideration of this procedure.

The VISX STAR S4-IR (Abbott Medical Optics, Inc., Santa Ana, CA, USA) excimer laser platform was used to perform all treatments. The default optical zone diameter was $6 \mathrm{~mm}$ (9 mm with transition zone); however, the treatment plan was individualized for each eye to achieve residual bed thickness of at least 350 microns. Physician adjustments were made, based on individual characteristics of subjects, such as age and amount of myopia correction.

All surgical procedures were performed under topical anesthesia. The corneal epithelium was removed with $20 \%$ alcohol for 20-40 seconds and the laser ablation was then applied, followed by accelerated CXL (Avedro Inc., Waltham, MA, USA). Vibex Rapid (Avedro Inc., Waltham, MA, USA) was instilled for 6-10 minutes followed by UV light irradiation of 3 minutes at $30 \mathrm{~mJ}$. Mitomycin C (0.02\%) was applied to the ablated stroma for 1 minute and washed thoroughly with balanced salt solution. A bandage contact lens (BCL) was then placed. 
The postoperative treatment regimen consisted of the combination of dexamethasone and moxifloxacin (Vigadexa, Alcon Laboratories, Inc., Fort Worth, TX, USA), 4 times a day for 10 days followed by fluorometholone with gradual tapering over 2 months. The BCL was removed after complete epithelialization, within a period of 4-10 days.

\section{Statistical analysis}

Data analysis was performed using the software SPSS for Windows version 17.0 (SPSS, Inc., Chicago, IL, USA). Normality of data samples was evaluated by the ShapiroWilk test and Q-Q plots. For normally distributed data, the Student's $t$-test for paired data was used for comparisons between the preoperative and postoperative data, whereas the Wilcoxon rank sum test was applied to assess the significance of such differences when data were not normally distributed. Differences were considered statistically significant when the associated $p$-value was $<0.05$. The standard graphs for reporting outcomes in refractive surgery were also plotted.

\section{Results}

\section{Preoperative characteristics}

Preoperative characteristics of 16 eyes were as follows: manifest refraction spherical equivalent (MRSE) was $-1.69 \pm 1.29 \mathrm{D}$ $(95 \% \mathrm{CI}-2.37,-1.00)$ and cylinder $-2.31 \pm 1.86 \mathrm{D}$ (95\% CI $-3.30,-1.32)$ (Figure 1). UDVA ( $\log$ MAR) was

A

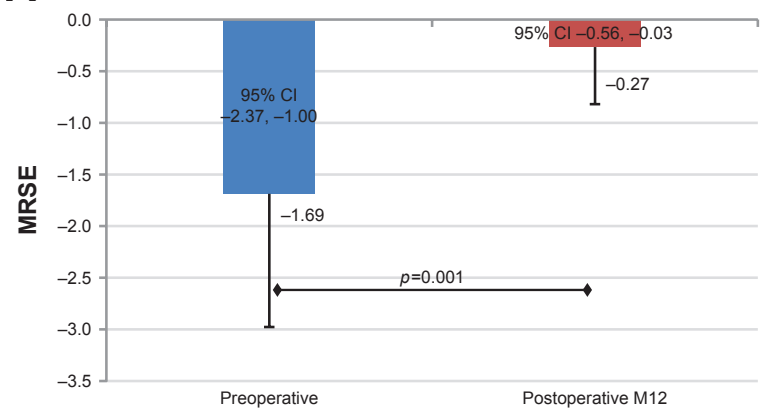

C

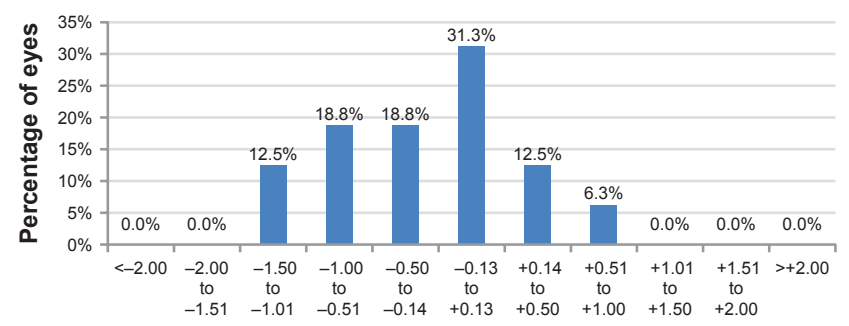

$0.56 \pm 0.33(95 \%$ CI $0.38,0.73)$ and CDVA (logMAR) was $0.16 \pm 0.10(95 \%$ CI $0.10,0.21)$ (Figure $3 \mathrm{~A}$ and B). Flat and steep keratectomy were $41.63 \pm 2.70 \mathrm{D}(95 \%$ CI $40.19,43.06)$ and $43.91 \pm 3.43 \mathrm{D}$ (mean $\pm \mathrm{SD})(95 \% \mathrm{CI} 42.08,45.73)$, respectively (Figure 4A and B).

\section{Visual and refractive outcomes}

A statistically significant improvement was observed at postoperative 12 months in MRSE and cylinder ( $p$-values: MRSE $=0.001$, cylinder $=0.004)$ with good refractive predictability and astigmatism accuracy (Figure 1A-D). A scatterplot of the attempted vs achieved MRSE at 12 months demonstrates good results (Figure 2). Likewise, statistically significant improvements were observed in both UDVA and CDVA 12 months postoperatively compared to the preoperative levels ( $p$-values: UDVA $=0.001$; CDVA $=0.007$ ) (Figure 3A and B). Efficacy was good, with $81.25 \%$ of eyes with postoperative UDVA of $20 / 32$ or better (Figure 3C). The majority (68.8\%) of eyes gained 1 or more lines of CDVA with $12.5 \%$ gaining 2 or more lines. There was no change in CDVA lines in $25 \%(4 / 16)$ eyes. No eye lost $\geq 2$ lines of CDVA (Figure 3D). One eye, which had severe keratitis, lost 1 line of CDVA, which subsided after adequate treatment, and the CDVA restored to preoperative levels of $20 / 40$ by the subsequent, 18 -month visit (corresponding to no loss in lines of CDVA at 18 months).

B

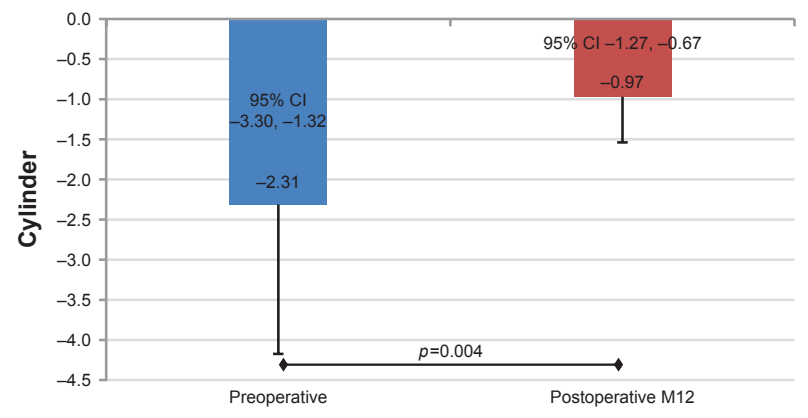

D

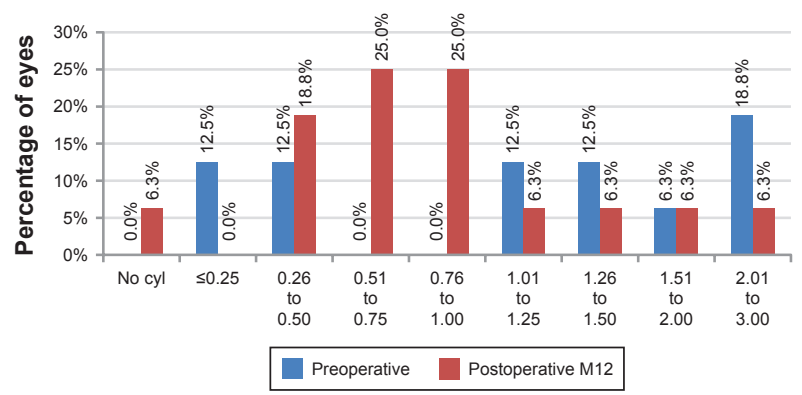

Figure I Histograms representing refractive outcomes of high-resolution wavefront-guided photorefractive keratectomy + corneal cross-linking in ectasia eyes. Note: (A) MRSE, (B) cylinder, (C) spherical equivalent refractive accuracy, (D) refractive astigmatism accuracy.

Abbreviation: MRSE, manifest refraction spherical equivalent. 


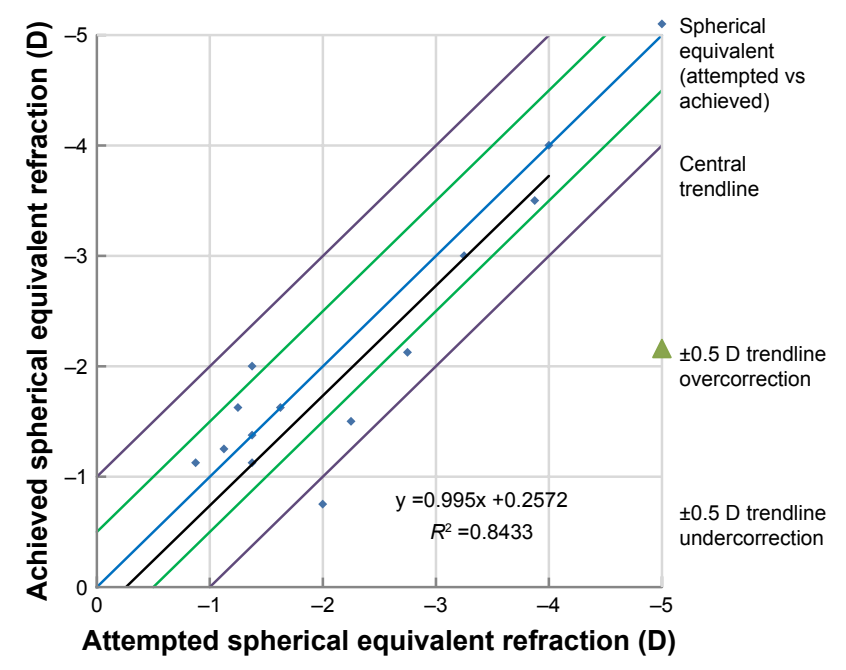

Figure 2 Scatterplot showing the relationship between the attempted and achieved spherical equivalent correction.

\section{Keratometric outcomes}

At postoperative 12 months, both flat and steep Ks were significantly reduced as compared to the preoperative level ( $p$-values; flat $\mathrm{K}=0.001$, steep $\mathrm{K}<0.001$ ) (Figure 4A and $\mathrm{B}$ ).

\section{HOAs}

A lowering trend was seen in the postoperative measurements of primary coma, primary trefoil, coma-like root mean square (RMS), trefoil-like RMS, and total RMS HOA, measured at

A

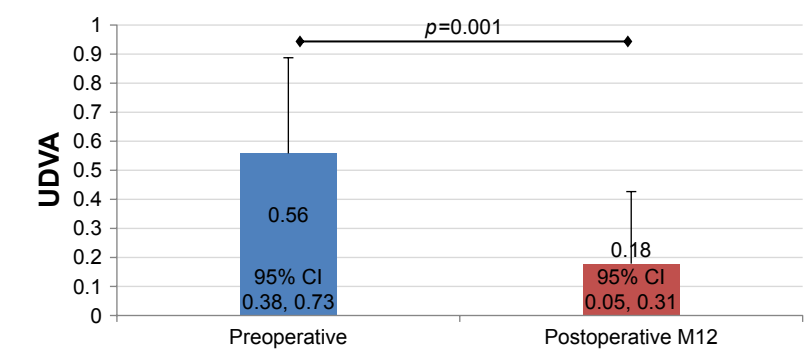

C

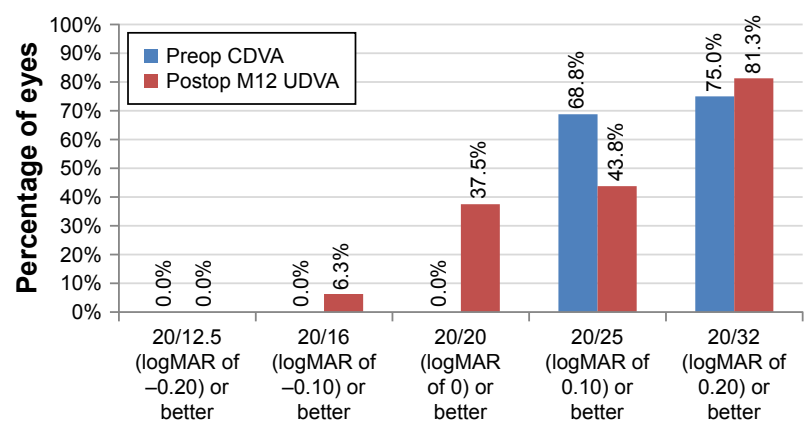

a pupil size of 4, 5, and $6 \mathrm{~mm}$ (Tables 1-3). Figure 5 demonstrates the preoperative and postoperative corneal topography axial power map with polar Zernike coefficients in one of the keratoconus eyes.

\section{Discussion}

In this study, we analyzed the outcomes of HRWG PRK (using a new-generation Hartmann-Shack aberrometer) + CXL, in ectasia eyes and found good improvement in aberrometry and statistically significant improvements in visual acuity, refraction, and keratectomy (Figures 1, 3, and 4) at postoperative 1 year, which are consistent with the findings reported by Shaheen et al, ${ }^{13}$ using the same aberrometer after WG PRK or LASIK in aberrated eyes. While Shaheen et al performed WG PRK in post-CXL keratoconus eyes, the current study evaluated the outcomes of combined WG PRK with CXL in keratoconus/post-LASIK ectasia eyes. Like the current study, Shaheen et al also documented statistically significant improvement in mean MRSE, visual acuity, and keratectomy at postoperative 6 months in keratoconus eyes that underwent WG PRK. This validates the potential of the WG PRK to provide enhanced visual function, particularly in aberrated eyes.

HOAs play an important role in reducing the quality of vision of ectasia eyes. After standard CXL for progressive

\section{B}

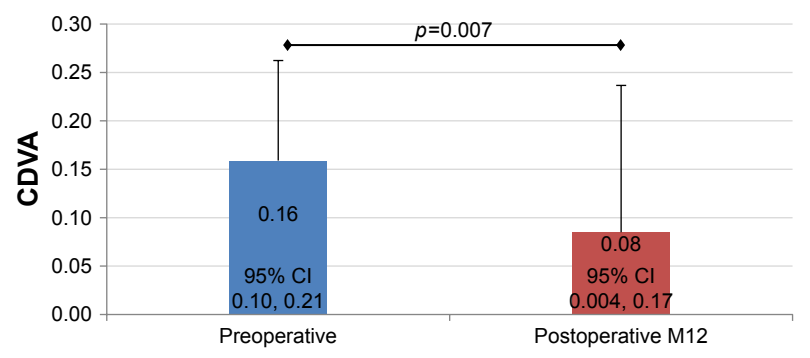

D

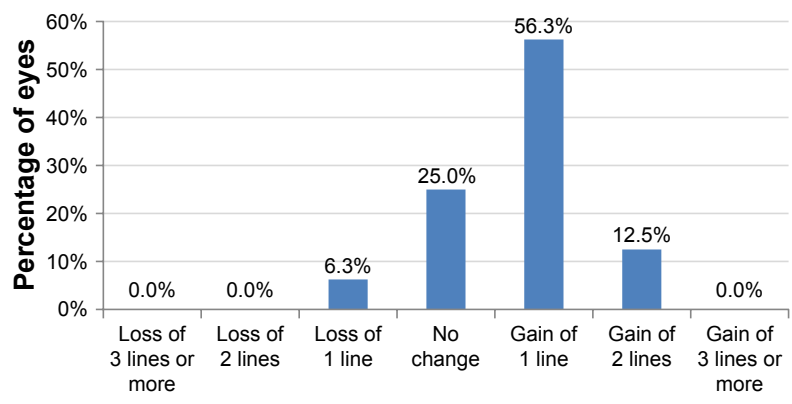

Figure 3 Histograms representing visual outcomes of high-resolution wavefront-guided photorefractive keratectomy + corneal cross-linking in ectasia eyes. Note: (A) UDVA, (B) CDVA, (C) efficacy chart, (D) safety chart.

Abbreviations: CDVA, corrected distance visual acuity; UDVA, uncorrected distance visual acuity. 
A

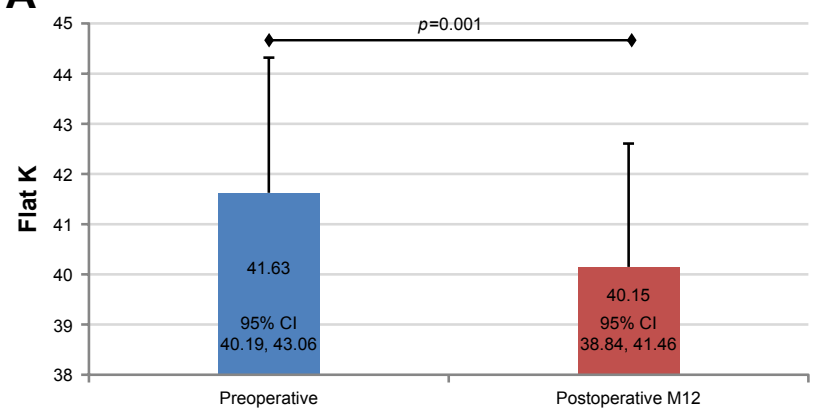

B

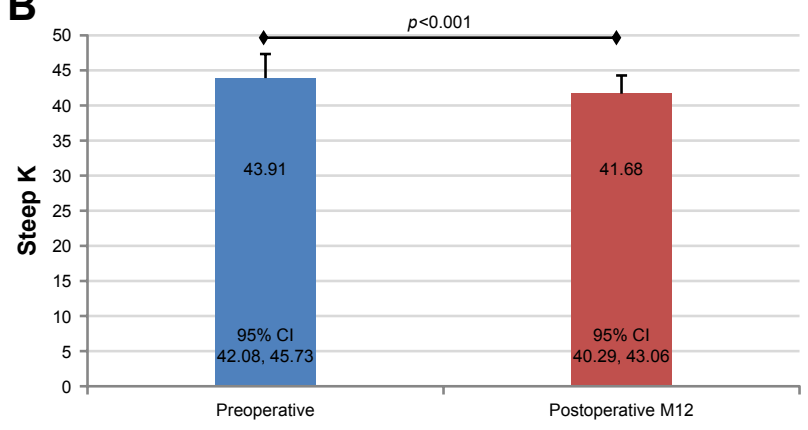

Figure 4 Histograms representing keratometric outcomes of high-resolution wavefront-guided photorefractive keratectomy + corneal cross-linking in ectasia eyes. Note: (A) Flat K, (B) steep K.

keratoconus, the HOAs have been reported to remain largely unchanged ${ }^{14}$ in the current study involving simultaneous HRWG PRK + CXL, a lowering trend was observed in ocular HOAs along with the consistent improvements in visual acuity, refraction, and keratectomy. Likewise, Shaheen et al also documented reductions in primary coma RMS, trefoil RMS, primary spherical aberrations, and HOA RMS, measured at a 6-mm pupil; however, none of these was statistically significant in keratoconus eyes. In addition to $6 \mathrm{~mm}$, we found a declining trend in the HOAs at 4 and $5 \mathrm{~mm}$ pupils as well; however, statistical significance was observed only for primary trefoil and trefoil-like RMS (4 mm pupil). Post hoc power calculations on the HOA RMS data from this pilot study indicate that larger sample sizes may reach statistical significance across all pupil sizes.

It is important to note that meaningful comparisons of the current study outcomes to published literature related to TG laser treatment are difficult given the significant differences in the magnitude of corneal ectasia (from early to advanced keratoconus) across studies. ${ }^{6,9,13,15}$ In addition, the TG ablations are centered on the corneal vertex and encompass the whole cornea, whereas WG ablations are centered on the pupil; therefore, it may be futile to compare the current study results with literature.

Refractive predictability is of significant importance following refractive surgery. In the current study, $87.5 \%$ eyes

Table I Preoperative and postoperative HOAs $4 \mathrm{~mm}(\mathrm{~N}=16)$

\begin{tabular}{llll}
\hline Type of HOAs & \multicolumn{1}{l}{ Preoperative } & Postoperative & p-value \\
\hline Primary coma RMS & $0.26 \pm 0.20$ & $0.21 \pm 0.20$ & 0.260 \\
Primary trefoil RMS & $0.14 \pm 0.12$ & $0.09 \pm 0.07$ & 0.027 \\
Primary spherical aberrations & $0.03 \pm 0.12$ & $0.03 \pm 0.1 \mathrm{I}$ & 0.652 \\
Coma-like RMS & $0.27 \pm 0.20$ & $0.21 \pm 0.20$ & 0.256 \\
Trefoil-like RMS & $0.14 \pm 0.12$ & $0.09 \pm 0.07$ & 0.026 \\
Spherical-like RMS & $0.07 \pm 0.09$ & $0.07 \pm 0.09$ & 0.501 \\
HOA RMS & $0.34 \pm 0.23$ & $0.27 \pm 0$ & 0.143
\end{tabular}

Note: Data presented as mean \pm SD.

Abbreviations: HOAs, higher-order aberrations; RMS, root mean square. were within $\pm 1.0 \mathrm{D}$ of attempted correction postoperatively. Although refractive predictability is subject to change over time, these values are much higher than the corresponding value of $45.5 \%$ reported by Shaheen et al following PRK in a group of previously cross-linked keratoconus eyes. ${ }^{13}$ One possible explanation for this difference is that in the Shaheen et al study the maximum ablation depth was limited to $50 \mu \mathrm{m}$; if the maximum ablation depth was more than $50 \mu \mathrm{m}$, a residual myopia was targeted. In contrast, the treatment plan in the current study was targeted to achieve residual bed thickness of at least $350 \mu \mathrm{m}$. Together with the different maximum ablation targets, the mean preoperative MRSE of the subjects in Shaheen et al study (mean -3.43 D) was higher than the current study (mean -1.69 D). As such, the refractive predictability was poorer in the Shaheen et al study. In addition, combined (simultaneous) PRK and CXL may produce better outcomes than sequential PRK and CXL because of minimization of potential superficial scarring associated with PRK and reduced haze formation as documented by Kanellopoulos in TG PRK and CXL. ${ }^{16}$ The potential disadvantage of simultaneous procedures could be a hyperopic shift due to CXL-associated flattening of the cornea. This disadvantage can be overcome by adjusting the treatment on an individualized basis.

Several surgical procedures are being used for the management of corneal ectasia; however, the best surgical approach

Table 2 Preoperative and postoperative HOA 5 mm ( $\mathrm{N}=12)$

\begin{tabular}{llll}
\hline Type of HOAs & Preoperative & Postoperative & p-value \\
\hline Primary coma RMS & $0.36 \pm 0.34$ & $0.25 \pm 0.17$ & 0.236 \\
Primary trefoil RMS & $0.21 \pm 0.17$ & $0.14 \pm 0.11$ & 0.083 \\
Primary spherical aberrations & $0.01 \pm 0.08$ & $0.02 \pm 0.10$ & 0.932 \\
Coma-like RMS & $0.36 \pm 0.34$ & $0.25 \pm 0.17$ & 0.233 \\
Trefoil-like RMS & $0.21 \pm 0.17$ & $0.14 \pm 0.11$ & 0.080 \\
Spherical-like RMS & $0.07 \pm 0.05$ & $0.07 \pm 0.07$ & 1.000 \\
HOA RMS & $0.46 \pm 0.36$ & $0.35 \pm 0.21$ & 0.211 \\
\hline
\end{tabular}

Note: Data presented as mean \pm SD.

Abbreviations: HOA, higher-order aberration; RMS, root mean square. 
Table 3 Preoperative and postoperative HOAs 6 mm (N=8)

\begin{tabular}{|c|c|c|c|}
\hline Type of HOAs & Preoperative & Postoperative & p-value \\
\hline Primary coma aberrations & $0.58 \pm 0$ & $0.38 \pm 0.33$ & 0.253 \\
\hline Primary trefoil aberrations & $0.33 \pm 0.29$ & $0.21 \pm 0.14$ & 0.192 \\
\hline Primary spherical aberrations & $0.00 \pm 0.11$ & $-0.02 \pm 0.12$ & 0.639 \\
\hline Coma-like aberrations & $0.59 \pm 0.6$ & $0.39 \pm 0.33$ & 0.257 \\
\hline Trefoil-like aberrations & $0.33 \pm 0.28$ & $0.22 \pm 0.14$ & 0.187 \\
\hline Spherical-like aberrations & $0.09 \pm 0.06$ & $0.09 \pm 0.09$ & 0.912 \\
\hline HOA RMS & $0.73 \pm 0.67$ & $0.52 \pm 0$ & 0.231 \\
\hline
\end{tabular}

Note: Data presented as mean \pm SD.

Abbreviations: HOA, higher-order aberration; RMS, root mean square.

should be determined based on the state of progression of the disease. ${ }^{17}$ Appropriate surgical option may delay the need for corneal transplantation in these patients. In patients with early, progressive keratoconus with a clear central cornea, CXL can reduce the risk of progression; ${ }^{18,19}$ however, if the patient is contact lens intolerant, $\mathrm{CXL}$ alone may not address the problem. For such cases, customized (either TG or WG) PRK with adjunctive CXL can be considered. ${ }^{3,7}$ In moderate keratoconus cases, if the patient has poor visual acuity because of the increased HOAs, HRWG PRK with CXL would be the best option. ${ }^{13}$ In advanced corneal ectasia with highly irregular anterior corneal surface, wavefront measurements may not be repeatable. In such cases, TG
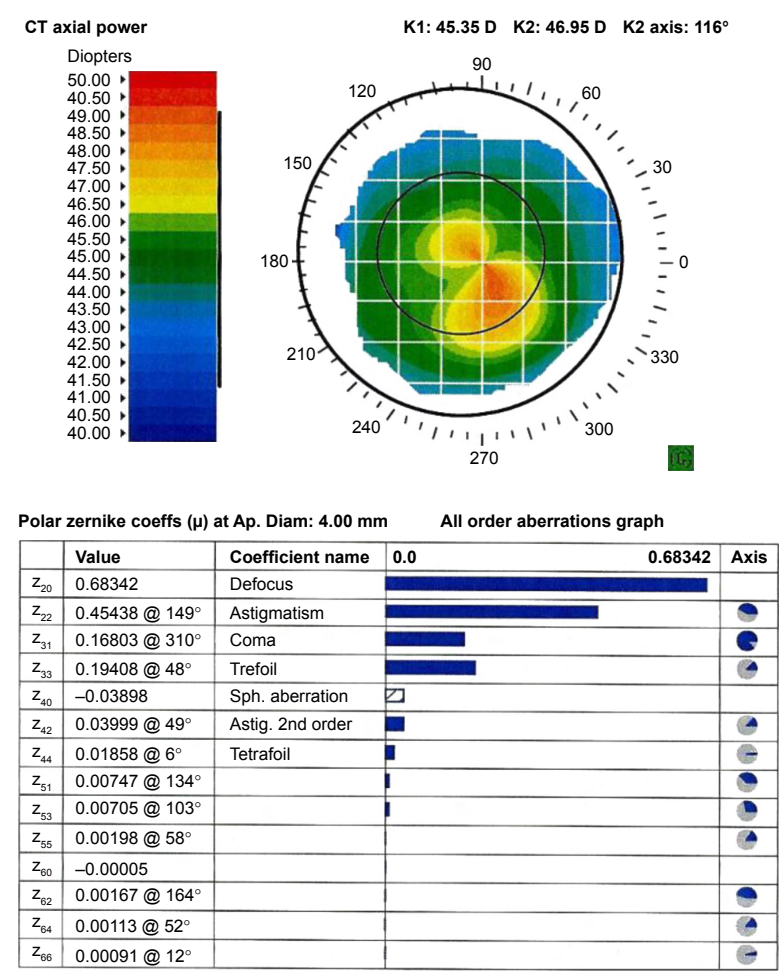

PRK with CXL remains an excellent option. Intrastromal corneal ring segments implantation is an alternative option for patients with thinner corneas. ${ }^{20}$

The main limitations of this study are the limited sample size of highly aberrated eyes and the short-term follow-up of 1 year. However, in a pilot study aimed at evaluating the possible role of WG ablation with CXL in irregular astigmatism, statistically significant improvements in visual acuity, refraction, and keratectomy are encouraging. Further studies with a larger sample size and longer follow-up would be of profound interest. CXL is known to halt progression and flatten the cornea over several years, ${ }^{21-23}$ which in the case of irregular astigmatism may be considered an advantage and not a concern. In our opinion, even short follow-up studies with a small number of patients like this one or the Shaheen et al study with only six months of follow-up ${ }^{13}$ deserve a careful look because we are speaking of a condition that was untreatable until few years ago.

\section{Conclusion}

The results of HRWG PRK in ectasia eyes using newgeneration Hartmann-Shack aberrometer in this pilot study are promising and provide surgeons with another tool in
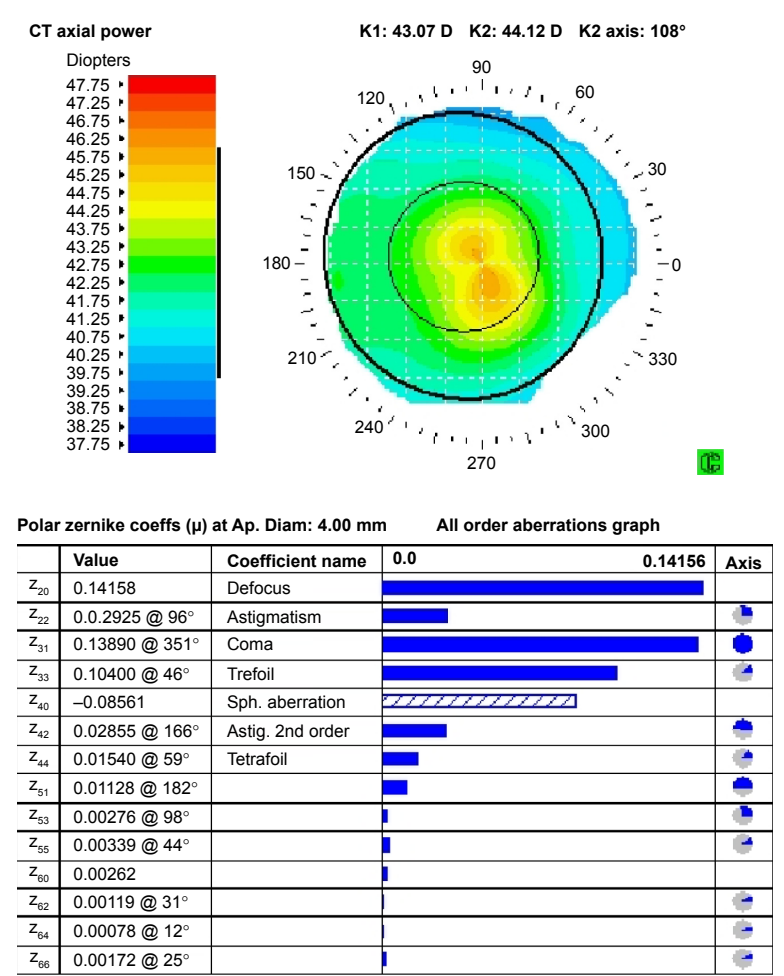

Figure 5 Preoperative and post-HRWG PRK + CXL corneal topography axial power map and polar Zernike coefficients, as recorded by iDesign software (iDesign, Abbott Medical Optics, Inc., Santa Ana, CA, USA) in one of the keratoconus ${ }^{\mathrm{TM}}$ eyes.

Notes: As evident, there is significant topographic improvement following HRWG PRK + CXL. Zernike coefficients, particularly, astigmatism, coma, and trefoil demonstrate considerable improvement. Overall, total root mean square error reduced from 0.94 preoperatively to 0.43 at postoperative 12 months.

Abbreviations: CXL, corneal cross-linking; HRWG, high-resolution wavefront-guided; PRK, photorefractive keratectomy. 
the armamentarium to improve vision in keratoconus/postLASIK ectasia with a high degree of refractive predictability. For the advanced keratoconus, even a high-dynamic range aberrometer may not be able to capture reproducible wavefront data. In such eyes, TG laser ablation remains an excellent option.

\section{Acknowledgments}

Raman Bedi, MD (IrisARC - Analytics, Research and Consulting, Chandigarh, India) and Jan Beiting (Wordsmith Consulting, Cary, North Carolina, USA) provided research, statistics, and editorial assistance in the preparation of this manuscript. Abbott Medical Optics Inc., Santa Ana, CA, USA, provided an unrestricted research grant.

\section{Disclosure}

GET is a consultant to AMO. The other authors report no conflicts of interest in this work.

\section{References}

1. Lin DT, Holland S, Tan JC, Moloney G. Clinical results of topographybased customized ablations in highly aberrated eyes and keratoconus/ ectasia with cross-linking. J Refract Surg. 2012;28(11 Supp1): S841-S848.

2. Cennamo G, Intravaja A, Boccuzzi D, Marotta G, Cennamo G. Treatment of keratoconus by topography-guided customized photorefractive keratectomy: two-year follow-up study. J Refract Surg. 2008;24(2): 145-149.

3. Kymionis GD, Portaliou DM, Diakonis VF, et al. Management of post laser in situ keratomileusis ectasia with simultaneous topography guided photorefractive keratectomy and collagen cross-linking. Open Ophthalmol J. 2011;5:11-13.

4. Lin DT, Holland SR, Rocha KM, Krueger RR. Method for optimizing topography-guided ablation of highly aberrated eyes with the ALLEGRETTO WAVE excimer laser. J Refract Surg. 2008;24(4):S439-S445.

5. Shetty R, D'Souza S, Srivastava S, Ashwini R. Topography-guided custom ablation treatment for treatment of keratoconus. Indian $J$ Ophthalmol. 2013;61(8):445-450.

6. Alessio G, L'Abbate M, Sborgia C, La Tegola MG. Photorefractive keratectomy followed by cross-linking versus cross-linking alone for management of progressive keratoconus: two-year follow-up. Am J Ophthalmol. 2013;155(1):54.e51-65.e51.

7. Kanellopoulos AJ, Binder PS. Management of corneal ectasia after LASIK with combined, same-day, topography-guided partial transepithelial PRK and collagen cross-linking: the athens protocol. J Refract Surg. 2011;27(5):323-331.

Clinical Ophthalmology

\section{Publish your work in this journal}

Clinical Ophthalmology is an international, peer-reviewed journal covering all subspecialties within ophthalmology. Key topics include: Optometry; Visual science; Pharmacology and drug therapy in eye diseases; Basic Sciences; Primary and Secondary eye care; Patient Safety and Quality of Care Improvements. This journal is indexed on Submit your manuscript here: http://www.dovepress.com/clinical-ophthalmology-journal
8. Sakla H, Altroudi W, Munoz G, Albarran-Diego C. Simultaneous topography-guided partial photorefractive keratectomy and corneal collagen crosslinking for keratoconus. J Cataract Refract Surg. 2014; 40(9):1430-1438.

9. Tuwairqi WS, Sinjab MM. Safety and efficacy of simultaneous corneal collagen cross-linking with topography-guided PRK in managing low-grade keratoconus: 1-year follow-up. J Refract Surg. 2012;28(5): 341-345.

10. Smadja D, De Castro T, Tellouck L, et al. Wavefront analysis after wavefront-guided myopic LASIK using a new generation aberrometer. $J$ Refract Surg. 2014;30(9):610-615.

11. Schallhorn S, Brown M, Venter J, Teenan D, Hettinger K, Yamamoto H. Early clinical outcomes of wavefront-guided myopic LASIK treatments using a new-generation hartmann-shack aberrometer. J Refract Surg. 2014;30(1):14-21.

12. Teenan D. Improving refractive results with wavefront-guided technology. Cataract and Refractive Surgery Today Europe 2012. Accessed October 1, 2012.

13. Shaheen MS, El-Kateb M, Hafez TA, Pinero DP, Khalifa MA. Wavefront-guided laser treatment using a high-resolution aberrometer to measure irregular corneas: a pilot study. J Refract Surg. 2015;31(6): 411-418.

14. Wisse RP, Gadiot S, Soeters N, Godefrooij DA, Imhof SM, van der Lelij A. Higher-order aberrations 1 year after corneal collagen crosslinking for keratoconus and their independent effect on visual acuity. $J$ Cataract Refract Surg. 2016;42(7):1046-1052.

15. Fadlallah A, Dirani A, Chelala E, Antonios R, Cherfan G, Jarade E. Non-topography-guided PRK combined with CXL for the correction of refractive errors in patients with early stage keratoconus. $J$ Refract Surg. 2014;30(10):688-693.

16. Kanellopoulos AJ. Comparison of sequential vs same-day simultaneous collagen cross-linking and topography-guided PRK for treatment of keratoconus. J Refract Surg. 2009;25(9):S812-S818.

17. Jhanji V, Sharma N, Vajpayee RB. Management of keratoconus: current scenario. Br J Ophthalmol. 2011;95(8):1044-1050.

18. Hafezi F, Kanellopoulos J, Wiltfang R, Seiler T. Corneal collagen crosslinking with riboflavin and ultraviolet A to treat induced keratectasia after laser in situ keratomileusis. J Cataract Refract Surg. 2007; 33(12):2035-2040.

19. O'Brart DP, Kwong TQ, Patel P, McDonald RJ, O'Brart NA. Longterm follow-up of riboflavin/ultraviolet A $(370 \mathrm{~nm})$ corneal collagen cross-linking to halt the progression of keratoconus. Br J Ophthalmol. 2013;97(4):433-437.

20. Vazirani J, Basu S. Keratoconus: current perspectives. Clin Ophthalmol. 2013;7:2019-2030.

21. Kymionis GD, Grentzelos MA, Liakopoulos DA, et al. Long-term follow-up of corneal collagen cross-linking for keratoconus - the Cretan study. Cornea. 2014;33(10):1071-1079.

22. O'Brart DP, Patel P, Lascaratos G, et al. Corneal cross-linking to halt the progression of keratoconus and corneal ectasia: seven-year follow-up. Am J Ophthalmol. 2015;160(6):1154-1163.

23. Raiskup F, Theuring A, Pillunat LE, Spoerl E. Corneal collagen crosslinking with riboflavin and ultraviolet-A light in progressive keratoconus: ten-year results. J Cataract Refract Surg. 2015;41(1):41-46.

PubMed Central and CAS, and is the official journal of The Society of Clinical Ophthalmology (SCO). The manuscript management system is completely online and includes a very quick and fair peer-review system, which is all easy to use. Visit http://www.dovepress.com/ testimonials.php to read real quotes from published authors. 